\title{
Tuning the excited state of photoactive building blocks for metal-templated self- assembly.
}

\begin{abstract}
The reaction of 2,2':4,4":4',4"'-quaterpyridyl (qtpy), with $\mathrm{d}(6)$ ruthenium(II) (Ru(II) ), and rhenium(I) $(\operatorname{Re}(\mathrm{I}))$ metal centers has been investigated. The pendant pyridyl groups on the products have also been methylated to produce a second series of complexes containing coordinated Meqtpy(2+). The absorption spectra of the complexes are dominated by intraligand and charge-transfer bands. The ruthenium(II) complexes display broad unstructured luminescence consistent with emission from a $\mathrm{Ru}(\mathrm{d}) \rightarrow \operatorname{diimine}\left(\pi^{*}\right)$ manifold in acetonitrile solutions. In aqueous solutions, their emissions are weaker and the lifetimes are shorter. This effect is particularly acute for complexes incorporating coordinated dipyridylpyrazine, dppz, ligands. Although the emission of the ruthenium(II) complexes containing Meqtpy(2+) is generally shorter than their qtpy analogs, it is notable that solventdependent effects are much less intense. The rhenium(I) complexes also display broad unstructured luminescence but, compared with the ruthenium(II) systems, they have a relatively short lifetime in acetonitrile. Electrochemical studies reveal that all of the $\mathrm{Ru}$ (II) complexes display chemically reversible metal-based oxidations. $\operatorname{Re}(\mathrm{I})$ complexes only display irreversible metal-based oxidations. In most cases, the reduction processes were not fully chemically reversible. The electrochemical and optical studies reveal that the nature of the lowest excited state of these complexes--particularly, the systems incorporating dppz--is highly dependent on the nature of the coordinated ligands. Calculations indicate that, although the excited state of most of the complexes is centered on the qtpy or Meqtpy(2+) ligands, the excited state of the complexes containing dppz ligands is switched away from the dppz by qtpy methylation. A crystallographic study on one of the dicationic ruthenium(II) structures reveals that it forms an inclusion complex with benzene.
\end{abstract}

Keyword: Ruthenium; Luminescence; Density functional theory; Electrochemistry; Complexes as ligands. 